\title{
Ab-Initio Calculations of Structural, Electronic, and Optical Properties of $\mathrm{Cd}_{1-x} \mathrm{Mn}_{x} \mathrm{Te}$
}

\author{
Bimal Kumar Sarkar, Member, IACSIT
}

\begin{abstract}
We have reported ab-initio calculations of the electronic, structural and linear optical properties of $\mathrm{Cd}_{1-x M n x T e}$ compounds. Ab-initio calculations are performed in the framework of full potential linearized-augmented plane-wave plus local orbitals $\left(\mathrm{FP}-\mathrm{LAPW}+\mathrm{l}_{0}\right)$ method based on the density functional theory (DFT) within the generalized gradient approximation (GGA). Murnaghan's equation of state (EOS) is used for volume optimization by minimizing the total energy with respect to the unit cell. The linear optical properties such as dielectric function, reflectivity, and refractive index are obtained. In the plots of the imaginary part of the complex dielectric function, the absorption threshold shifts toward higher energy with the increase in Mn contents.
\end{abstract}

Index Terms-Ab initio calculations, density-functional theory, equations of state, optical properties, dielectric function.

\section{INTRODUCTION}

A great deal of attention has been attributed to the study of diluted magnetic semiconductor due of their potential applications in a wide spectrum of optoelectronic devices, photovoltaic solar cells, laser screen materials and various luminescence devices, etc. [1]. $\mathrm{Cd}_{1-x} \mathrm{Mn}_{x}$ Te is one of them, especially important due to variation of its electronic and optical properties by changing the $\mathrm{Cd}: \mathrm{Mn}$ doping. In this paper we have reported the ab-initio calculations for the investigation of electronic, structural, and optical properties of $\mathrm{Cd}_{1-x} \mathrm{Mn}_{x} \mathrm{Te}$ alloys using full potential linearized-augmented plane-wave plus local orbitals $\left(\right.$ FP-LAPW $\left.+1_{0}\right)$ method based on the density functional theory (DFT) within the generalized gradient approximation (GGA) [2].

\section{Computational Details}

FP-LAPW+lo method as implemented in the Wien $2 \mathrm{k}$ code [3] with GGA parameterized by Perdew, Burke and Ernzerhof (PBE) [4] is employed to deal with the exchange and correlation effects. The equilibrium structural parameters are carried out by optimizing total energy with respect to the unit cell volume using Murnaghan's equation of state [5]. The calculations were done with $R_{M T} k_{\max }=7$, to achieve energy eigen value convergence. $R_{M T}$ is the smallest radius of the muffin-tin (MT) spheres and $k_{\max }$ is the maximum value of the wave vector. The wave function has been expanded inside the atomic spheres with the maximum value of the

Manuscript received February 21, 2014; revised April 26, 2014.

Bimal Kumar Sarkar is with the Galgotias University, India (e-mail: bks@physics.org.in). angular momentum $l_{\max }$ as 10 . The irreducible Brillouin zone (BZ) has been decomposed into a matrix of $10 \times 10 \times 10$ Monkhorst-Pack $k$-points [6]. The iteration procedure is continued with total energy and charge convergence to $0.0001 \mathrm{Ry}$ and $0.001 \mathrm{e}$, respectively [7], [8].

\section{RESULTS AND DISCUSSIONS}

The $\mathrm{Cd}_{1-x} \mathrm{Mn}_{x}$ Te has a cubic symmetry with space group F-43M. The total energy is optimized with respect to the unit-cell volume by fitting Murnaghan's equation of state [5]:

$$
E_{T}(V)=\left[\frac{B_{0} V}{B_{0}{ }^{\prime}} \frac{\left(V_{0} / V\right)^{B_{0}{ }^{\prime}}}{B_{0}{ }^{\prime}-1}+1\right]+E_{0}-\frac{V_{o} B_{o}}{B_{0}{ }^{\prime}-1}
$$

For $x=0,0.25,0.5,0.75,1$, Table I shows the energy minimization occuring for $a_{0}=6.617,6.548,6.487,6.422$ and $6.371 \AA$, which agree well with the experimental values [9]-[11].

TABLE I: EEQUilibrium LATTICE CONSTANT $A_{0}(\AA)$, Bulk Modulus B $B_{0}$ (GPA) AND ITS PRESSURE DERIVATIVE $\mathrm{B}_{0}{ }^{\prime}$ OF $\mathrm{CD}_{1-X} \mathrm{MN}_{X} \mathrm{TE}$

\begin{tabular}{ccccccc}
\hline \multicolumn{3}{c}{$a_{0}(\AA)$} & \multicolumn{3}{c}{$\mathrm{B}_{0}(\mathrm{GPa})$} & $\mathrm{B}_{0}{ }^{\prime}$ \\
\cline { 2 - 7 } & \multicolumn{7}{c}{ Present } & Others & Present Others & Presen Others \\
\hline 0.0 & 6.617 & $6.56[9]$ & 37.02 & - & 3.58 & - \\
0.25 & 6.548 & $6.481[11]$ & 34.92 & $44.50[11]$ & 4.54 & $6.40[11]$ \\
0.5 & 6.487 & $6.354[10]$ & 35.61 & $47.10[10]$ & 4.64 & $4.58[10]$ \\
0.75 & 6.422 & $6.32[8]$ & 37.78 & $49.94[8]$ & 4.99 & $7.46[8]$ \\
& & $6.39[11]$ & & & & \\
1.0 & 6.371 & $6.26[9]$ & 36.12 & - & 3.58 & - \\
\hline
\end{tabular}

Hence well-defined structural properties are sufficient for further study of electronic and optical properties. The equilibrium lattice constants are used to calculate the density of states (DOS) and electronic band structures for $\mathrm{Cd} 1-x \mathrm{Mn}_{x} \mathrm{Te}$ (shown in Fig. 1 and Fig. 2). The zero of the energy scale is set at the Fermi level $\left(\mathrm{E}_{\mathrm{F}}\right)$. The energy band structures are calculated along the directions containing high symmetry points of the first Brillouin zone, namely $\mathrm{W} \rightarrow \mathrm{L} \rightarrow \Gamma \rightarrow \mathrm{X} \rightarrow \mathrm{W} \rightarrow \mathrm{K}$. Each member of $\mathrm{Cd}_{1-x} \mathrm{Mn}_{x}$ Te demonstrates the existence of the valence band maximum and conduction band minimum at the same symmetry point. This confirms the direct energy gap between the top of the valence band and the bottom of conduction band at $\Gamma$ point. These plots provide a qualitative explanation of the atomic and orbital origins of the different band states. 
From the DOS plots we observe that near the Fermi level, the upper part of the valence band possesses Mn-3d and Te-5p characteristics.

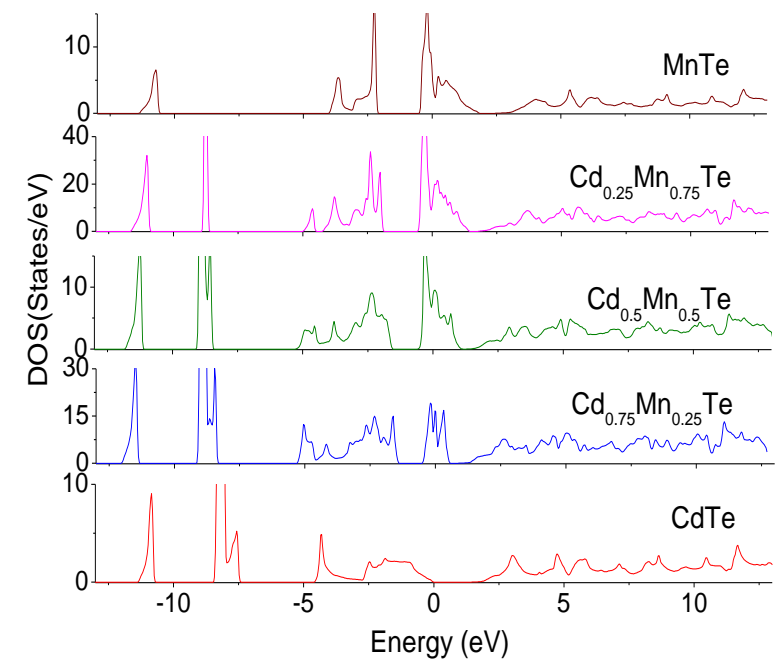

Fig. 1. Electron density of states for $\mathrm{Cd}_{1-x} \mathrm{Mn}_{x} \mathrm{Te}$.

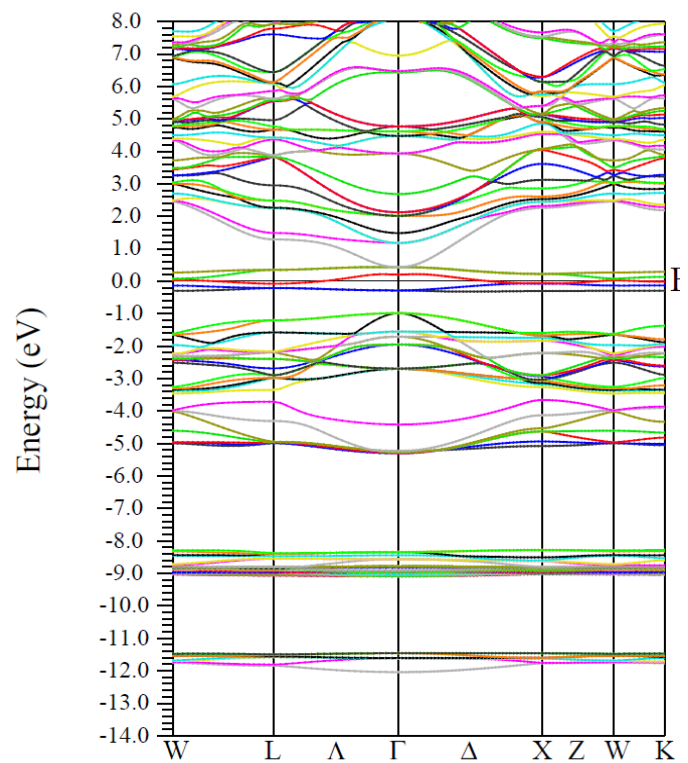

Fig. 2. Electronic band structure for $\mathrm{Cd}_{0.75} \mathrm{Mn}_{0.25} \mathrm{Te}$.

The first structure in the total DOS is small and centered at around $-11.19 \mathrm{eV},-11.28 \mathrm{eV},-11.58 \mathrm{eV}$ and $-11.87 \mathrm{eV}$ for $x=1.0,0.75,0.5$ and 0.25 respectively. This structure arises from the Te s states and it corresponds to the lowest lying band with the dispersion in the region around the $\Gamma$ point in the Brillouin zone. The next structure appears at $-8.20 \mathrm{eV}$, $-8.18 \mathrm{eV},-8.11 \mathrm{eV}$ and $-8.01 \mathrm{eV}$ for $x=0.75,0.5,0.25$ and 0.0 respectively. It is an attribute of $\mathrm{Cd} \mathrm{d}$ states with some $\mathrm{p}$ states of the Te atoms and occupies largest number of states with flat bands clustered between $-9.0 \mathrm{eV}$ and $-8.2 \mathrm{eV}$. Less dispersion of these bands results in sharp peaks. There is a wide spread in DOS in the energy range of $-5.0 \mathrm{eV}$ and zero energy for these compounds. The peaks in this energy interval arise from the Te $\mathrm{p}$ states partially mixed with $\mathrm{Cd} \mathrm{s}$ states and they contribute to the upper Valence Band. Above the Fermi level, the feature in the DOS originate mainly from the $s$ and $p$ states of Cd partially mixed with little of Te d states. Band width of valence band as determined from the width of the peaks in DOS dispersion below Fermi level equal to $12.42 \mathrm{eV}, 12.13 \mathrm{eV}, 12.17 \mathrm{eV}$ for $x=0.75,0.5,0.25$, respectively. The results showing valence band width minimum for $\mathrm{Cd}_{0.75} \mathrm{Mn}_{0.25} \mathrm{Te}$, clearly indicate that the wave function for $\mathrm{Cd}_{0.75} \mathrm{Mn}_{0.25} \mathrm{Te}$ is more localized than that for others. This is in consistence with the fact that when the atomic number of the anion increases, a material becomes non-polar covalent with valence band states being more localized. The dielectric function $\varepsilon(\omega)$ can describe the interaction of photons with electrons in the form of linear response of the system to electromagnetic radiation [12]. Dielectric function comprises of the imaginary part $\varepsilon_{2}(\omega)$ and the real part $\varepsilon_{1}(\omega)$. The momentum matrix elements between the occupied and unoccupied wave functions within the selection rules play the main role for the determination of $\varepsilon_{2}(\omega)$. The expression for $\varepsilon_{2}$ is given by

$$
\begin{aligned}
\varepsilon_{2}(\omega) & =\frac{V e^{2}}{2 \pi \hbar m^{2} \omega^{2}} \int d^{3} k\left|\left\langle k n|p| k n^{\prime}\right\rangle\right|^{2} f(k n) \\
& \times\left[1-f\left(k n^{\prime}\right)\right]\left(E_{k n}-E_{k n^{\prime}}-h \omega\right)
\end{aligned}
$$

where $\hbar \omega$ is the energy of the incident photon, $p$ is the momentum operator, $|k n\rangle$ is the eigenfunction with eigenvalue $E_{k n}$ and $f(k n)$ is the Fermi distribution function.

The real part $\varepsilon_{1}(\omega)$ of the dielectric function follows the Kramer-Kroning relations and can be expressed in terms of $\varepsilon_{2}(\omega)$ as follows

$$
\varepsilon_{1}(\omega)=1+\frac{2}{\pi} \int_{0}^{\infty} \frac{\varepsilon_{2}\left(\omega^{\prime}\right) \omega^{\prime} d \omega^{\prime}}{\omega^{\prime 2}-\omega^{2}}
$$

The variation of the dielectric functions, $\varepsilon_{2}(\omega)$ and $\varepsilon_{1}(\omega)$ of $\mathrm{Cd}_{1-x} \mathrm{Mn}_{x} \mathrm{Te}$ as function of photon energy is displayed in Fig. 3 and Fig. 4 respectively, in the range of $0-14 \mathrm{eV}$.

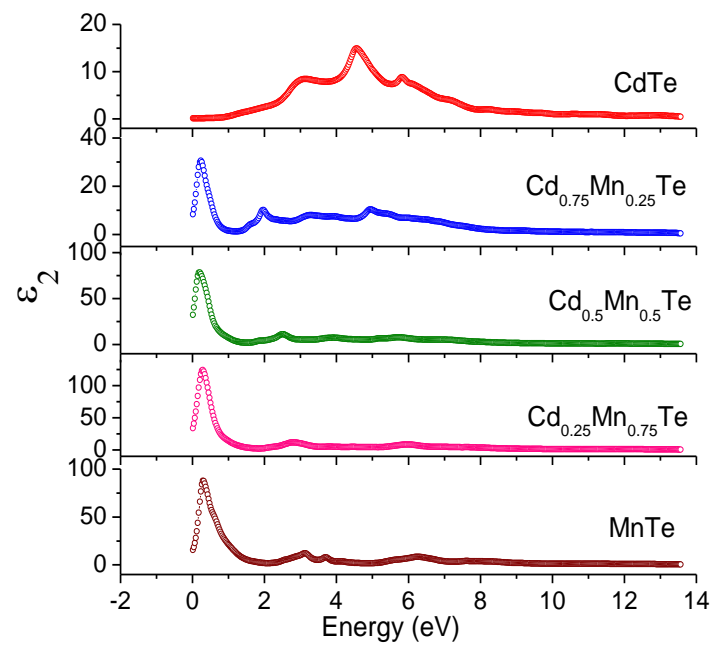

Fig. 3. The variation of the dielectric functions, $\varepsilon_{2}$ of $\mathrm{Cd}_{1-\mathrm{X}} \mathrm{Mn}_{\mathrm{X}} \mathrm{Te}$ as function of photon energy.

It is observed that the imaginary part of the complex dielectric function, the absorption threshold shifts toward higher energy with the increase in Mn contents. With the knowledge of the imaginary part $\varepsilon_{2}(\omega)$ and the real part $\varepsilon_{1}(\omega)$ of the dielectric function, one can calculate different optical properties. The following equations are used for the 
calculation of refractive index $\mathrm{n}$ and reflectivity $\mathrm{R}[13]-[15]$ :

$$
\begin{gathered}
n(\omega)=\frac{1}{\sqrt{2}} \sqrt{\varepsilon_{1}^{2}(\omega)+\varepsilon_{2}^{2}(\omega)+\varepsilon_{1}} \\
R(\omega)=\left|\frac{\sqrt{\varepsilon(\omega)}-1}{\sqrt{\varepsilon(\omega)}+1}\right|^{2}
\end{gathered}
$$

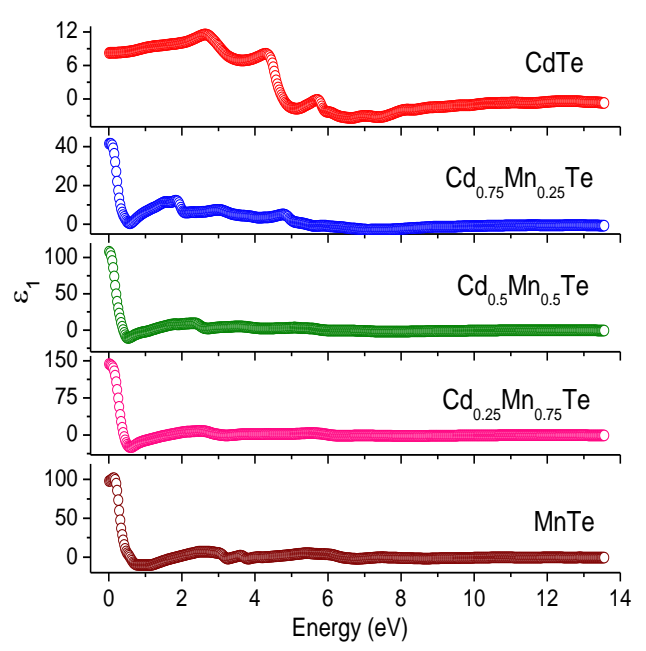

Fig. 4. The variation of the dielectric functions, $\varepsilon_{1}$ of $\mathrm{Cd}_{1-x} \mathrm{Mn}_{x} \mathrm{Te}$ as function of photon energy.

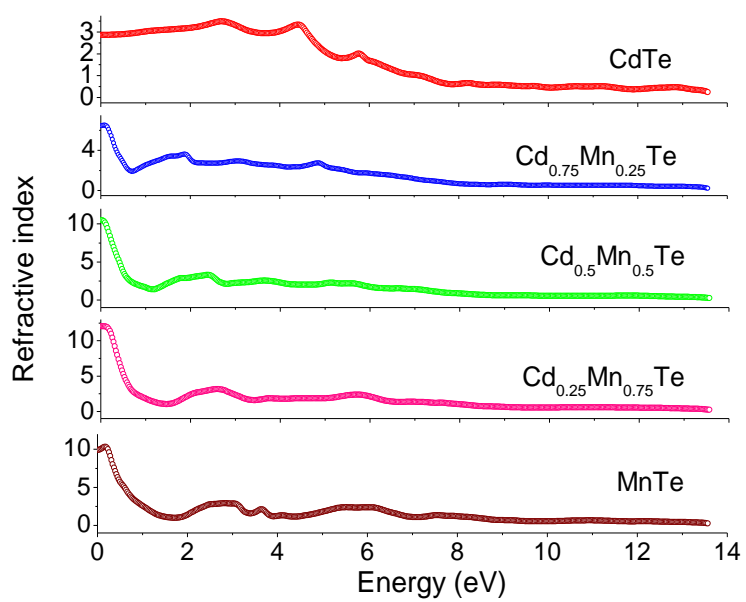

Fig. 5. The variation of the refractive index, $(n)$ of $\mathrm{Cd}_{1-x} \mathrm{Mn}_{x} \mathrm{Te}$ as function of photon energy.

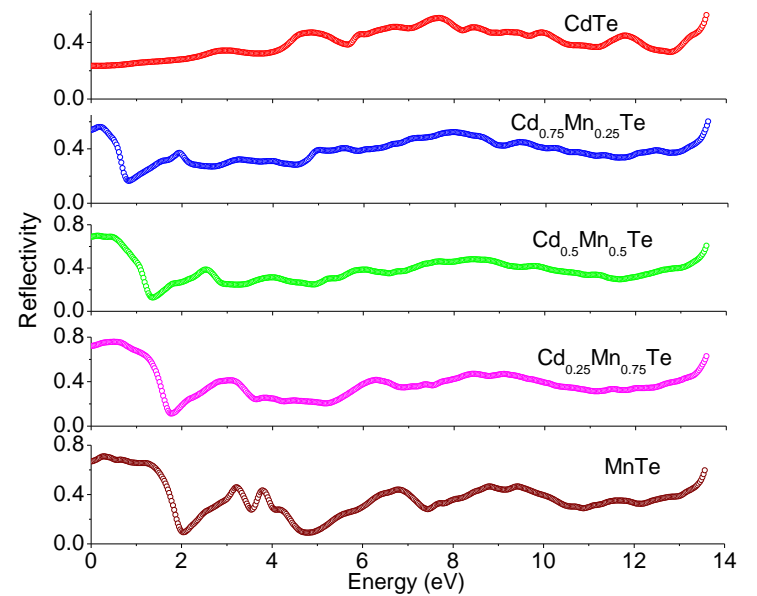

Fig. 6. The variation of the reflectivity $(R)$ of $\mathrm{Cd}_{1-x} \mathrm{Mn}_{x} \mathrm{Te}$ as function of photon energy.
The calculated optical parameters, viz., refractive index $n(\omega)$ and reflectivity $R(\omega)$ are shown in Fig. 5 and Fig. 6 respectively. It is clear from the reflectivity spectrum that $\mathrm{CdTe}$ possesses more transmitting nature than $\mathrm{Mn}$ doped compounds.

\section{CONCLUSION}

The structural, electronic, and optical properties of Cd 1-XMn $\mathrm{M}_{\mathrm{X}}$ Te have been studied with FP-LAPW + lo method in the framework of density functional theory. The quantities such as band structure, dielectric constants, and refractive index were obtained. The generalized gradient approximation (GGA) was considered for the exchange and correlation effects calculations. The band structure of all compounds confirms the direct energy gap between the top of the valence band and the bottom of conduction band at $\Gamma$ point. In the linear optical response, the absorption threshold shifts toward higher energy with the increase in Mn contents.

\section{ACKNOWLEDGMENT}

We acknowledge the support in part by the Department of Science and Technology through computing resources provided by the High Performance Computing Facility at the Inter University Accelerator Centre, New Delhi. The author (BKS) is thankful to Galgotias University for its support.

\section{REFERENCES}

[1] Y. C. Zhang, W. W. Chen, and X. Y. Hu, "Controllable synthesis and optical properties of zn-doped cds nanorods from single-source molecular precursors," Cryst. Growth Des, vol. 7, pp. 580-586, February 2007.

[2] S. Z. Karazhanov, P. Ravindran, U. Grossner, A. Kjekshus, H. Fjellvåg, and B. G. Svensson, "Coulomb correlation effects in zinc monochalcogenides," J. Appl. Phys. vol. 100, pp. 043709, August 2006.

[3] P. Blaha, K. Schwarz, G. K. H. Madsen, D. Kvasnicka, and J. Luitz, "Computer code WIEN2K (Vienna University of Technology, 2002), improved and updated Unix version of the original," in P. Blaha, K. Schwarz, P. Sorantin, S. B. Rickey, ed., Comput. Phys. Commun., vol. 59, pp. 399-415, June 1990.

[4] J. P. Perdew, K. Burke, and M. Ernzerhof, "Generalized gradient approximation made simple," Phys. Rev. Lett. vol. 77, pp. 3865-3868, October 1996.

[5] F. D. Murnaghan, "On the theory of the tension of an elastic cylinder," in Proc. Natl. Acad. Sci., USA, vol. 30, pp. 382-384, December 1944.

[6] H. J. Monkhorst and J. D. Pack, "Special points for brillouin-zone integrations," Phys. Rev. B., vol. 13, pp. 5188-5192, June 1976.

[7] A. S. Verma, S. Sharma, B. K. Sarkar, and V. K. Jindal, "First principles study on the elastic and electronic properties of $\mathrm{CdX}(\mathrm{X}=\mathrm{S}$, Se and Te)," AIP Conf. Proc., vol. 1393, pp. 237-239, February 2011.

[8] S. A. Touat, F. Litimein, A. Tadjer, and B. Bouhafs, "The spin effect in zinc-blende $\mathrm{Cd} 0.5 \mathrm{Mn} 0.5 \mathrm{Te}$ and $\mathrm{Zn} 0.5 \mathrm{Mn} 0.5 \mathrm{Te}$ diluted magnetic semiconductors: FP-LAPW study," Physica B, vol. 405, pp. 625-631, January 2010.

[9] S. H. Wei and A. Zunger, "Total-energy and band-structure calculations for the semimagnetic $\mathrm{Cd}_{1-\mathrm{x}} \mathrm{Mn}_{\mathrm{x}} \mathrm{Te}$ semiconductor alloy and its binary constituents," Phys. Rev. B, vol. 35, pp. 2340-2365, February 1987.

[10] A. E. Merad, M. B. Kanoun, and S. Goumri-Said, "Ab initio study of electronic structures and magnetism in ZnMnTe and CdMnTe diluted magnetic semiconductors," J. Magn Magn. Mater, vol. 302, pp. 536-542, July 2006.

[11] A. Balzarotti, M. Czyzyk, A. Kisiel, N. Motta, M. Podgorny, and M. Zmnel-Starnawska, "Local structure of ternary semiconducting random solid solutions: Extended x-ray-absorption fine structure of $\mathrm{Cd}_{1-\mathrm{x}} \mathrm{Mn}_{\mathrm{x}}$ Te," Phys. Rev. B, vol. 30, pp. 2295-2298, August 1984.

[12] R. Khenata, A. Bouhemadou, M. Sahnoun, A. H. Reshak, H. Baltache, and M. Rabah, "Elastic, electronic and optical properties of $\mathrm{ZnS}, \mathrm{ZnSe}$ 
and ZnTe under pressure," Comput. Mater. Sci. vol. 38, pp. 29-38, November 2006.

[13] Y. Shen and Z. Zhou, "Structural, electronic, and optical properties of ferroelectric KTa1/2Nb1/2O3 solid solution," J. Appl. Phys. vol. 103, pp. 074113, April 2008.

[14] M. Dadsetani and A. Pourghazi, "Optical properties of strontium monochalcogenides from first principles," Phys. Rev. B vol. 73, pp. 195102, May 2006

[15] F. Wooten, Optical Properties of Solids, Academic, New York, 1972, pp. $67-81$.

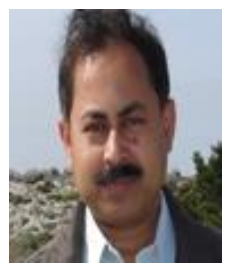

Bimal Kumar Sarkar was born on January 2, 1961, in Kolkata, India. He graduated in physics at Jadavpur University and electrical engineering at Institute of Engineers (India). He did his Ph.D. on the topic photoacoustic spectroscopic estimation of thermal and optical properties of solids at Indian Association for the Cultivation of Science, India. He pursued a postdoctoral fellowship at the Department of Chemistry, National Sun Yat-sen University, Taiwan. Afterward he visited in research positions at NSYSU, Taiwan, Czech Technical University, Slovak Technical University etc.
Now he is a professor of physics at Galgotias University, India with activity in research and teaching. At present, he is involved in the field of computational materials and computational biology. His research interest is ab initio study of the structural, lattice dynamics, electronic, elastic, thermodynamic, optical properties of solids. First-principles calculations based on density functional theory (DFT) are performed to study the structural and electronic properties of solids. Also he is doing computational work based on information theory for the investigation of network architecture of genome controlled signal transduction circuit. 